\title{
Las agencias de viajes receptivas cubanas: una quimera entre un modelo societario y un modelo cooperativo
}

\author{
Isnel Martínez Montenegro \\ Magíster en Administración de Empresas, Universidad de Matanzas «Camilo Cienfuegos», \\ Profesor de derecho Mercantil Universidad de Matanzas «Camilo Cienfuegos»
}

Sumario: I. Introducción. II. Bases teóricas que demuestran que en las agencias de viajes existe la posibilidad de una quimera entre los dos modelos. III. Elementos comunes y contrapartidas de ambos modelos. IV. Ideas conclusivas. V. Bibliografía.

Resumen: Las empresas de intermediación turística en los países receptores, adquieren la forma de compañías mercantiles. En Cuba la intermediación turística se desarrolla a través de las agencias de viajes, y la ley prohíbe otras modalidades de intermediadores. En este sentido una propuesta de quimera entre el modelo societario existente y el cooperativo pudiera contribuir al desarrollo de la comercialización de los productos turísticos, logrando una mejor gestión si se logra, además, que funcionen incorporando los principios del cooperativismo moderno.

Palabras clave: Agencias de viajes, intermediación turística, derecho del turismo.

Abstract: Tourist intermediation companies in the recipient countries take the form of commercial companies. In Cuba tourism intermediation it takes place through travel agencies, the law prohibits other forms of intermediators. In this regard a proposal for a chimera between the existing corporate model and the cooperative could contribute to the development of marketing of tourism products, achieving better management if it is also operating incorporating the principles of cooperation achieved.

Keywords: Travel agencies receptive, tourist intermediation, tourism law. 


\section{Introducción}

La industria turística mantiene una estrecha relación con otros sectores de la economía nacional cubana, en la medida que comienza a elevar su productividad, otros como los servicios, la construcción, la agricultura, el transporte, el ocio y la artesanía, también empiezan a crecer inmediatamente debido al empuje que le impregnan los visitantes extranjeros y nacionales.

Signados todos, por la compleja red de hoteles, restaurantes, agencias de viajes, tour operadores, medios de transporte, atracciones, servicios públicos, entre otros, que se componen integrando un sistema para que el negocio turístico se proyecte y desarrolle de modo eficaz y satisfactorio.

En el año 2015 el destino turístico de Varadero y la capital de la isla se registran como los destinos turísticos más visitados, aunque se aprecia un aumento en la práctica de grupos de turistas que se complacen de otras opciones tradicionales y ecológicas. La diversificación de las ofertas ha sido provocada por la voluntad de transformar a la industria turística cubana, en no solo, un destino de sol y playa, sino también en vender el producto a través de otras excelentes atracciones como son: la práctica del turismo ecológico, la pesca deportiva, los deportes náuticos y el submarinismo; además del turismo de ciudad, donde se aprovechan los vetustos y conservados centros patrimoniales de las antiguas villas.

Varadero posee una vasta red hotelera y cuenta con una extensa red extrahotelera compuesta por tres Marinas, un campo de golf, un aeropuerto internacional, varias agencias de viajes ${ }^{1}$, una amplia red gastronómica y de recreación. La red extrahotelera desempeña un papel decisivo en el proceso de comercialización, y aunque se ha consolidado como un destino de sol y playa, existen otras propuestas como son: las actividades ecológicas, y del acervo cultural e histórico de la localidad.

En tal sentido, se aprecia que este sector, como ningún otro, depende de un sistema de apoyo integrado por puertos, aeropuertos y carreteras, así como, de la energía y del entorno en general, los cuales presentan grandes oportunidades para mejorar si funcionan de manera conjunta. De modo, que se afirma la necesidad no solo, de la integración de los factores, sino además, de su desarrollo productivo por separado (Suñol, 2008: 60).

1 Receptivos del destino turístico de Varadero: Viajes Cubanacán, Havanatur, Paradiso, Ecotur, Cubatur, Cubamar y Gaviota Tours Varadero. 
Asimismo, queda demostrada la importancia de las empresas de intermediación turística en la unificación de los diferentes factores que conforman la red de comercialización del producto turístico, debido en gran medida a que son entidades que organizan servicios turísticos diversos (viajes combinados, excursiones, representación de otras agencias de viajes) y facilitan, a nivel mundial y nacional, su mercantilización, junto con los servicios ofrecidos por otras empresas (fundamentalmente, reservas de plazas en medios de transporte de viajeros y de alojamientos turísticos), sirviendo de enlace principal en muchas ocasiones, y necesariamente imprescindibles entre la oferta y la demanda turística.

En el caso específico de la intermediación turística, poseen un carácter de actividad turística empresarial libre y reglamentada, sometida al otorgamiento de una previa autorización, ejercida de forma profesional y habitual, consistente en la mediación, comercialización y organización de servicios turísticos (Sanz, 2005: 121). En el caso de esta modalidad empresarial en Cuba, se propone un modelo enfocado al desarrollo de la figura cooperativa, como quimera a la realización hasta este momento, de un modelo societario, que no se ajusta a la realidad empresarial que se despliega por los receptivos del destino turístico, ni de otros actores que intervinen en el proceso de comercialización del producto.

De esta manera no sería ingenuo proponer un modelo de cooperativas no agropecuarias si éstas poseen el objetivo general que taxativamente se establece en la Ley cubana de produccir bienes y prestar servicios, y en su objeto social (...) la producción, prestación de servicios o actividad de comercialización (López, 2001: 232), coincidente con las funciones que desempeñan estos receptivos en la comercialización de sus productos turísticos. En este sentido, es que dirigimos las investigación a establecer caracteres legales que permitan orientar al operador jurídico y a los agentes hacia una tipología empresarial de las agencias de viajes receptivas - dentro de los marcos de la política del turismo nacional- conforme a la una gestión cooperativa, estableciendo presupuestos para una quimera entre un modelo cooperativo y societario en el desarrollo y comercialización de los productos turístico, en Cuba.

\section{Bases teóricas que demuestran que en las agencias de viajes existe la posibilidad de una quimera entre los dos modelos}

Las agencias de viajes son empresas que se dedican profesionalmente al ejercicio de actividades de mediación u organización de ser- 
vicios turísticos, con tres funciones básicas: asesora, mediadora y productora (López, 2001: 236). Las mismas no tienen limitado su ámbito territorial de actuación y pueden extender su actividad a un gran número de servicios turísticos.

De esta forma la ocupación principal de este tipo de empresa turística es la de realizar la intermediación entre el prestador del servicio y el turista, convirtiéndose en la fuerza de venta más importante y concentrando en un solo lugar, la gama de servicios y productos que ofrecen (Albert, 1995: 76). Asimismo se convierte en asesora cuando orientan al cliente en lo referente y desde su punto de vista profesional con respecto al producto más conveniente a elegir dentro de un gran grupo de opciones; y la última función la desarrolla como integradora o vendedora de servicios y productos turísticos propios, o sea, cuando según los criterios de la doctrina iberoamericana (García, 1999; Gómez, 1975; Martínez, 1999; Kemelmajer, 2006; Bech, 2007) en los viajes de todo incluido que reciben el nombre de paquetes y se organizan desde una perspectiva integral de los servicios que brindan las agencias de viajes, acoplados en un nuevo servicio que se comercializa y establece en un precio para ese mismo producto (Pérez, 1996: 89).

El primer elemento que nos permitiría posteriormente pensar en una compatibilidad de la utilización del modelo cooperativo es el fundamento legal para este tipo de empresa en $\mathrm{Cuba}^{2}$, donde su definición se corresponden directamente con los que establece la ley española ${ }^{3}$, aunque esta última, sí delimita la categorización según el criterio de la magnitud y operación, demarcando los sujetos responsables frente al consumidor según la organización y comercialización del producto, o sea, los paquetes turísticos que ofrecen.

En una clasificación empresarial abarcadora de ambas sintonías legales, las agencias de viajes mayoristas trabajan diferentes destinos, proyectan, elaboran y organizan toda clase de servicios turísticos, para su venta posterior a través de las agencias de viajes minoristas, y uti-

2 Resolución Conjunta 1/1998, de 6 de diciembre, de las agencias de viajes de Cuba, en su artículo primero inciso a) establece que las Agencias de viajes nacionales se definen como las personas jurídicas que se encuentren inscritas en el Registro Nacional de Agencias de Viajes y que tengan en su objeto social, la realización de actividades que consistan en la mediación entre los viajeros y aquellas personas jurídicas autorizadas a prestar servicios a estos de diferente naturaleza a las actividades propias de Agencias de Viajes.

3 Real Decreto 20/1997, de las agencias de viajes de la Comunidad Valenciana; Decreto 81/ 2012, de 22 de mayo, de las agencias de viajes de la Comunidad Autónoma del País Vasco; Decreto 301/2002, de 17 de diciembre, de la agencias de viajes y centrales de reservas de la Comunidad Autónoma de Andalucía. 
lizando para ello su red de ventas en el exterior. Con la característica fundamental de que no ofrecen directamente sus productos al usuario o consumidor del producto turístico. En cambio las agencias de viajes minoristas venden directamente a los consumidores y clientes los productos de las mayoristas o sus paquetes turísticos, y servicios individuales que proyecten, elaboren y organicen. Finalmente las agencias mayoristas-minoristas que pueden simultanear las actividades de las dos anteriores categorías.

Asimismo es oportuno precisar que existen otros criterios comunes para clasificar a este tipo de empresa de intermediación turística, y sin que sea el interés primordial de los autores, brevemente presentaremos los diferentes criterios ya que a partir del entendimiento de la figura y de su fucionamiento, es que el lector podrá adherirse a la propuesta de un funcionamiento cooperativo de los mismos en Cuba.

En este sentido se puden observar según el criterio de operación a la que se dedican:

Operadora como mayorista o mayorista-minorista. Operan sus propios viajes preparados para grupos numerosos o personas individuales, o los que otorga en preparación a las agencias grandes o pequeñas.

De igual manera prestadoras de servicios donde se limitan a ofertar alquiler de automóviles con o sin chofer, guías de turismo, o bien sólo actúan como representante de alojamientos y transportistas (Guevara, 2009: 39).

Con respecto al tipo de turistas que manejan las agencias de viajes receptivas pueden funcionar como empresas encargadas de organizar y manejar viajes y/o proporcionar ciertos servicios aislados al turista que proviene de un lugar distinto al de la localización de la agencia. Reconocidas también como agencias de incoming, prestan el servicio al turista que proviene de un entorno geográfico distinto al del sujeto intermediador, o sea, el ofrecido por un mayorista extranjero o tour operador (López, 2001: 239). Esta forma empresarial es la manera más común en que se proyectan las empresas de intermediación turísticas en Cuba, y es por ende el eje central de nuestra propuesta de actuación bajo un sistema cooperativo local o regional.

Las agencias de turismo de exportación o emisora tienen como particularidad que venden tanto servicios aislados como viajes organizados, generalmente por otras agencias, a turistas nacionales o extranjeros que viajen hacia el destino seleccionado. También conocidas como outgoing y sus funciones también son cercanas a las que analizamos en la presente investigación (Martínez, 2014: 41).

Un supuesto especial son la agencias de viajes de turismo receptivo y emisoras debido a que estas manejan simultáneamente viajes para tu- 
ristas dentro y en el exterior del país; las mismas se dedican a la actividad de emisión, pero disponen de sus receptivos propios para orientar a los clientes nacionales y extranjeros. La diferencia es mínima con las formas anteriores y pudieran perfectamente ser compatibles con esta propuesta.

Con relación al canal de distribución sería interesante analizar las agencias de viajes in-plant, que son pequeñas unidades o subordinaciones que se instalan en clientes con el objetivo de mejorar la calidad del servicio brindado, o sea, con estos establecimientos se aumenta el contacto con los clientes. Asimismo entendemos que en las condiciones en que se desarrolla el turismo cubano en la actualidad perfectamente podrían presentarse como una posibilidad viable para la proyección del sistema que se formula.

\section{Elementos comunes y contrapartidas de ambos modelos}

Es importante antes de comenzar con el acentamiento de los elementos establecer que la postura de la normativa cubana con respecto al tipo social de las agencias de viajes, es el de homogenizar la forma societaria que adquieren, según lo dispuesto como sociedad capitalista anónima.

En Cuba la sociedad mercantil puede definirse como la asociación voluntaria de personas que crean un fondo común, con ánimo de obtener un beneficio individual de carácter lucrativo, participando en el reparto de las ganancias que se obtengan. Las sociedades mercantiles, significan la forma de equiparar a la agrupación voluntaria de personas que buscan obtener un beneficio legítimo de inversiones a través de la aportación de capital, industria o bienes, como parte del perfeccionamiento de su actividad mercantil (Colectivo de Autores, 2001: 68).

De esta forma pudieramos observarlas desde dos puntos de vista:

Sociedad como contrato: contrato formal, legal y típico, de agrupación voluntaria de personas con personalidad jurídica propia; donde predomina la voluntad de los socios. Dos o más personas ponen en común bienes o industrias para realizar una actividad económica y obtener ganancias.

Sociedad como institución: persona jurídica que se crea con aporte patrimonial social; a la que el Estado le reconoce personalidad jurídica, derechos y obligaciones. Este ente jurídico ha de tener nacionalidad, domicilio, nombre y patrimonio, o sea, derechos y deberes. Vía externa de la sociedad. 
Al igual que los autores de la doctrina española (Vicent, 1988: 136; Broseta, 2008: 236) alegamos que la sociedad anónima es la sociedad mercantil, constituida intuitu pecuniae, con su capital social divido en acciones, que invisten a su titular con la condición de socio y estas son, a su vez, incorporadas a títulos de fácil transmisión, donde el accionista además gozan de la responsabilidad limitada frente a las deudas arrogadas por la compañía, y no responden por las deudas sociales adjudicadas frente a terceros.

Sin apartarnos de este camino pudieramos corresponder la compatibilidad existente entre este modelo societario con el cooperativo, las cooperativas vienen normalmente revestidas de la forma de una sociedad anónima o de una sociedad de responsabilidad limitada. La peculiar forma de asociación de responsabilidad limitada, que tuvo su mayor acogida en el medio rural, es hoy menos frecuente, aunque pudiera ser perfectamente aplicable al diseño de los receptivos turísticos cubanos. Ahora bien, por vía estatutaria - las entidades de integración cooperativa adquieren en este punto un protagonismo de primer orden- adoptan aquellas determinaciones propias del funcionamiento cooperativo (Montolio, 2001: 41).

En Mexico en el Código de Comercio las sociedades cooperativas fueron consideradas como una variante de las sociedades mercantiles y quedaron definidas de esa manera, con todas sus características generales tales como: el número de socios, el capital variable, su responsabilidad solidaria e ilimitada o limitada a una determinada suma menor igual o mayor que el capital social (Labriega, 1985: 24).

Esta misma tendencia de observar en su naturaleza jurídica a las cooperativas como sociedad la mantiene España y por ende es que se propone un modelo semejante al caso de estudio, fundándose la proyección en el argumento que Cuba mantiene la vigencia del Código de Comercio Español desde 1886 y su correspondiente modelo societario «en primer lugar» y "en segundo lugar» los modelos de funcionamiento de las agencias de viajes que analizamos desde sus propias modalidades es también semejante al español, aunque con algunas diferencias en cuanto algunas de las comunidades autónomas.

En este aspecto la Ley cubana no prevee la constitución de esta clase de sociedades. Por tales motivos se han visto en ocasiones los operadores forzados a tomar los modelos societarios y sus recursos brindados por empresas de tipo capitalistas, convirtiendo a los intermediadores en cualquiera de sus modalidades en accionista y la posibilidad de la gestión cooperativa e integrada de los receptivos, se ha desechado. De esta forma debemos apuntar que se niega la posibilidad que genera la intercooperación entre las diferentes agencia 
de viajes existentes, y aunque pudiera decirse que se vinculan en su comercialización no gozan del beneficio del sexto principio de la Declaración de la Alianza Cooperativa Internacional, sobre la identidad cooperativa.

Del mismo modo sería una intercooperación si se crea una estructura local en Varadero o en otro de los destinos turísticos donde interactuen varios receptivos, para facilitar las operaciones con mayor fortaleza en el entorno turístico y alcanzar los objetivos competitivos con mayor facilidad. Sería la mejor manera de realizar la función de intermediación de una manera más eficaz en un entorno cada vez más competitivo (Villa, 2000: 203).

De esta manera cuando nos acercamos al mercado cubano y a su constante lucha contra los diferentes factores externos e internos, iqué mejor solución que el uso del cooperativismo!, que están «seleccionadas por la evolución natural» de las especies económicas como empresas dotadas frente a las crisis, frente a las adversidades económicas, puesto que nacieron en ese medio natural y llevan siglos de supervivencia en el mismo. "Está en su ADN la lucha por la superación de las dificultades, no son empresas débiles que sucumben fácilmente ante la hostilidad de las crisis que producen los ciclos económicos. Sigue apuntando.... El resaltamiento de la importancia económica de las relaciones intercooperativas no es por ello nada nuevo. Desde la Conferencia Económica Mundial celebrada en Ginebra en mayo de 1927 se aprobó una resolución al efecto» (Divar, 2010: 269).

Visto de este modo solo nos queda revisar algunas de las posibles dificultades que tendrían que enfrentar los ejecutores de la propuesta, observadas en cierta medida en las cuestiones que diferencian un modelo societario y uno cooperativo en la doctrina cubana, y si asumimos como caso de estudio para el modelaje el que se gestiona en el destino de Varadero en Matanzas, Cuba.

Primeramente apuntamos el análisis a los presupuestos de la intermediación como actividad empresarial y fuente de empleo, aunque con los objetivos de lograr el desarrollo de las actividades autonómas en el territorio, ya que de esta forma no sería ilógico aplicar un modelo donde no solo, se aporta capital, sino trabajo de los asociados también, y donde estos se benefician de la gestión en su condición de cooperativistas y correspondan con su servicio en la toma de decisiones con voz y voto. Pensar en el desarrollo de un turismo sostenible y sustentable, basado en la gestión de la oferta en el turismo de sol y playa es imposible, este empeño solo se pudiera alcanzar si desde su misma comercialización se alcanza implicar a los diferentes factores que intervienen como actores en la gestión, que se sientan identificados con el colec- 
tivo y su gestión a través de la satisfacción de sus necesidades materiales y espirituales.

La fórmula se pudiera completar con los principios que se enarbolan en la Alianza Universal de Cooperativas, en función de ese modelo de gestión de los intermediarios turísticos, si se hacen corresponder con un aporte sustancial por parte de los intermediadores en su conjunto. Trazar a través de su aplicación el camino más flexible mediante el cual se logre moldear el modelo existente con los confines del cooperativismo, el papel de los diferentes participantes y la forma de distribuir los excedentes, convierten a la cooperativa y a su gestión en materia de intermediación diferente a otras empresas, y el cambio indiscutiblemente, puede beneficiar a la industria turística cubana que en ocasiones se afecta por las reclamaciones por costos de no calidad y por la ineficiente gestión de estos sujetos que mutan a un intermediador con contacto cero con el cliente.

Tres de los principios de la Ailianza que pueden aportar mayor fortaleza a la propuesta son los de educación, formación e información; cooperación entre cooperativas y el de interés por la comunidad. El primero aporta educación y formación a los socios, a los representantes elegidos, a los directivos y a los empleados para que puedan contribuir de forma eficaz al desarrollo de sus cooperativas, informan al gran público, especialmente a los jóvenes y a los líderes de opinión, de la naturaleza y beneficios de la cooperación. Sería un aporte importante para el desarrollo de la intermediación en la región con más fuerza en el turismo cubano, donde en ocasiones se ejercita el oficio sin contar con las herramientas necesarias y se desarrolla de manera ilegal; y al monopolio estatal pudiera bien corresponderle un intercambio de experiencias hasta el momento inexistente.

En el segundo de los citados es prácticamente uno de los elementos más importantes de la propuesta, debido a que a través de su desarrollo, se sirven los socios de una forma más eficaz que la establecida en el modelo societario actual, en primer lugar, porque se puede posibilitar y fortalecer el movimiento cooperativo trabajando conjuntamente mediante estructuras locales, nacionales, regionales e internacionales; cuestión esta última que tienen solo identificada los agentes pero materialmente no la tienen diseñada ni protegida, y menos aún, con los beneficios que pudiera brindar el modelo cooperativo.

El tercero y no menos importante en este análisis proporciona que asumiendo un modelo cooperativo estas agencias receptivas puedieran realizar sus funciones de intermediadores turísticos con la perspectiva de un aporte al desarrollo de sus comunidades, en este caso de estudio a la de Varadero, mediante políticas aprobadas por los so- 
cios y encamidadas al desarrollo local de un turismo más competitivo del que existe hoy en día, comercializado mayormente, en ofertas de sol y playa.

Son muchos los aspectos que reflejan la superioridad de un modelo cooperativo, aunque no es el objetivo asumirlo completamente sobre el modelo societario. En la actualidad los diferentes receptivos cubanos relacionan algunos de los elementos que abordamos en esta quimera y entre ellos se encuentran:

- Los socios participan directamente en la gestión y este elemento es catalizador de motivación, además de reflejarse en el principio analizado de educación, capacitación e información.

- Especial interés por el funcionamiento de este ente y por tanto existe un protagonismo por parte de los socios en la toma de decisiones, que puede hacerse corresponder con la efectividad en el proceso de comercialización del producto turístico.

- Fuente de empleo estable, poco abocadas al fracaso como empresa y aglutinadora de trabajadores identificados con el objeto social.

- No acumulación de riquezas, posibilidad de utilizar fondos en función de la comunidad que los rodea y este elemento puede ser catalizador de un turismo más efectivo, sustentable y sostenible, con parámetros de acogida superiores en un beneficio mutuo empresa-población.

- La cooperación se distingue de la competencia clásica del uno contra el otro, especializaría a cada una de las agencias de viajes con productos específicos y mercados de clientes, asi de esta manera estuvieran puntualizados en sus gestiones.

- En el caso Cuba existe salarios mínimos establecidos en tarifas salariales fijadas por la Ley Laboral que se verían limitadas si se asume el modelo, y esto puede permitir incentivar al trabajador, concientes de la ventaja de la figura del modelo cooperativo vigente, lo que indudablemente aumentará la eficiencia y la calidad en la producción o el servicio que prestan los receptivos.

\section{Ideas conclusivas}

La superficial aproximación que precede a la tipología de las empresas de intermediación turísticas en Cuba, representada por las agencias de viajes, y el breve análisis de los elementos de los modelos analizados 
con la integración de algunos de los principios del cooperativismo nos conduciría a concluir que:

La conceptuación empresarial de las agencias de viajes en Cuba es compatible aunque se encuentran legalmente contituídas en regímen societario, a la orientación del modelo cooperativo que le es connatural si analizamos las condiciones en que se desarrollan su estrategias de comercialización y de proyección empresarial. La influencia de la legislación española se hace en este punto manifiesta.

Lo cierto es que las ventajas de la limitación de la responsabilidad que brindan el modelo societario asumido por los intermediadores en Cuba, constituye una propuesta eficaz para todos aquellos esfuerzos que produzcan el desarrollo social con el fin de superar las reclamaciones al Estado cubano y a su patrimonio estatal socialista, creando una ficción jurídica y protegiendo este último, aunque no es menos cierto que existen elementos y principios del modelo cooperativo que pudieran contribuir de un modo más palpable e inter personae al desarollo de la industria turística cubana, de una manera sostenible y sustentable.

\section{Bibliografía}

AlBerT, I.: Gestión y técnicas de agencias de viajes, Universidad de Barcelona, Barcelona, 1995, p. 78.

$\mathrm{BECH}$, J. M.: La responsabilidad contractual de los organizadores y detallistas de viajes combinados, Universidad de Girona, Girona, 2007, pp.165-167.

Broseta, M.: Manual de Derecho Mercantil, Universidad de Valencia, Valencia, 1988, p. 143.

Colectivo de Autores.: Temas de Derecho Mercantil cubano, Editorial Félix Valera, La Habana, 2008, p. 68

DeCReto Ley 305.: Ley de Cooperativas no Agropecuaria, Gaceta Oficial, La Habana, 2012.

DIVAR, J.: «Las cooperativas ante la crisis económica», REVESCO, núm. 44, pp. 263-283.

GARCíA, M. P.: La responsabilidad contractual de las agencias de viaje, Editorial Montecorvo, Madrid, 1999, p. 143.

Gómez, J.: "Régimen jurídico del contrato de viaje combinado», Revista de Derecho Mercantil, núm.137, pp. 301-328.

Guevara, E.: Procedimiento para el análisis del producto opcionales en la agencia de viajes Gaviota Tours Varadero, Universidad de Matanzas "Camilo Cienfuegos», Matanzas, 2009, p.62.

KeMELMAJER, A. «El contrato de servicio turísticos. Su realidad en la jurisprudencia reciente», Revista Latinoamericana de Derecho, núm.5, pp. 97-101.

LABRIEGA, P.: La reforma de legislación Mercantil, Editorial Porrúa, Mexico, 1985, pp.84-86. 
LÓPEZ, F.: Factores condicionantes de la ventajas competitiva de los resultados de las agencias de viajes en España, Universidad de Barcelona, Barcelona, 2001, pp. 227-339

Martínez, P.: El contrato de viaje combinado (Antecedentes, Derecho Comparado, Estudio Normativo y Jurisprudencial), Universidad de Castilla-La Mancha, Cuenca, 1999.

MARTínez, I.: Procedimiento para el análisis del marco legal de las agencias de viajes en Varadero, Universidad de Matanzas «Camilo Cienfuegos», Matanzas, 2014, p. 42.

Montolio, J. "Capital Social y Cooperativas en los Países Escandinavos», REVESCO, pp. 263-283.

SANZ, C.: Régimen jurídico-administrativo de las empresas de intermediación turística, Universidad de Sevilla, Sevilla, 2005, p. 326.

Suñol, S. «Entorno competitivo para el desarrollo del turismo. Caso Puerto la Plata», Revista Ciencia y Sociedad, núm. 1, pp. 60-81.

Pérez, A.: El mundo de los agentes de viaje, Universidad de Barcelona, Barcelona, 1996, p.66.

Resolución CONJUNTA.: Reglamento de la Agencias de Viajes Nacionales, Sucursales y Representaciones de Agencias de Viajes Extranjeras de la República de Cuba, Gaceta Oficial, La Habana, 1998.

RodRIGO, U.: Derecho Mercantil, Editorial Marcial Pons, Madrid, 1997.

VICENT, F.: Derecho Mercantil, Universidad de Valencia, Valencia, 1988.

VILLA, E.: "La intercooperación de las sociedades cooperativas en la actividad educativa», REVESCO, núm. 1, pp. 42-53. 